\title{
Photo-induced Superparamagnetism In Trimetallic Coordination Nanoparticles
}

\author{
Daniela. Brinzei, Laure Catala, ${ }^{*}$ Corine Mathonière,${ }^{b}$ Wolfgang. Wernsdorfer, ${ }^{c}$ Alexandre Gloter, ${ }^{d}$ \\ Odile Stephan ${ }^{d}$ and Talal Mallah ${ }^{*}$ \\ a Institut de Chimie Moléculaire et de Matériaux d'Orsay, CNRS, Université Paris Sud, 91405 Orsay Cedex, \\ France. \\ Fax: (+33)(0)16915 47 54; Tel: (+33)(0)16915 47 49; \\ ${ }^{b}$ ICMCB, UPR CNRS 9048, Université Bordeaux 1, 87 avenue du Docteur Albert Schweitzer, F-33 608 Pessac \\ Cedex, France. \\ ${ }^{c}$ Institut Néel, CNRS, BP 166, 25 Avenue des Martyrs, 38042 Grenoble Cedex 9, France. \\ ${ }^{d}$ Laboratoire de Physique des Solides, UMR CNRS 8502, Université Paris-Sud, , 91405 Orsay Cedex, France.
}

\section{Supplementary Materials}

\section{$\underline{\text { Experimetal details }}$}

\section{Preparation}

To $100 \mathrm{~mL}$ of an aqueous solution containing $34.1 \mathrm{mg}$ of $\mathrm{CuCl}_{2} \cdot 2 \mathrm{H}_{2} \mathrm{O}, 47.5 \mathrm{mg} \mathrm{NiCl}{ }_{2} \cdot 6 \mathrm{H}_{2} \mathrm{O}$ and $4.44 \mathrm{~g}$ of PVP K30 $(\mathrm{ALDRICH}, \mathrm{Mw}=40000$ ) was added dropwise (under gentle stirring at room temperature) $100 \mathrm{~mL}$ aqueous solution containing $99.2 \mathrm{mg}$ of $\mathrm{K}_{4} \mathrm{Mo}(\mathrm{CN})_{8}$. A change of color to purple appears. The solution was left still one hour. Addition of $600 \mathrm{~mL}$ of acetone leads to the recovering of a polymeric-like violet compound that was centrifuged and dried under vacuum. A film can be obtained from simple evaporation of the solvent.

\section{Elemental Analysis : CuNiMo(CN) $)_{\mathbf{8}}(\mathbf{P y r})_{\mathbf{4}} \mathbf{. 5 9} \mathbf{H}_{\mathbf{2}} \mathrm{O}$}

Exp \% C 51,21; H 7.59; N 11,39; Mo 1,10; Ni 0,64; Cu 0,69

Calc \% C 51,92; H 8,12; N 11,48; Mo 1,43; Ni 0,87; Cu 0,95

Despite the uncertainty on the stoichiometry due to the highly diluted sample (less than $1 \%$ metal), a similar stoichiometry $0.95 \mathrm{Cu}: 1 \mathrm{Ni}: 1 \mathrm{Mo}(\mathrm{CN})_{8}$ was obtained on a bulk compound obtained without PVP. Furthermore, the magnetism is in good agreement before irradiation with this composition.

\section{Irradiation experiments}

Photomagnetic experiments were carried out with a Quantum Design MPMS-5S magnetometer working in the dc and ac modes. The two photomagnetic experiments described in this communication were performed with a $\mathrm{Kr}^{+}$laser coupled through an optical fiber directed into the squid cavity. They were performed at $5 \mathrm{~K}$ up to 5 Teslas for magnetization, in the 2-50K range with magnetic field of $100 \mathrm{G}$ (experiment A, figures 2 and S3) and in the 2-300K range with magnetic field of $5000 \mathrm{G}$ (experiment B, figure S2) in the dc modes. For experiment A, the susceptibilities in ac mode were measured at frequencies ranging from 10 to $1000 \mathrm{~Hz}$ with an ac field amplitude of 2 Oe and no dc field applied with in the $2-8 \mathrm{~K}$ range. For both experiments, the output of the fiber is situated to a distance of $5 \mathrm{~cm}$ from the sample. Coated nanoparticules were laid down on the sample holder. The diamagnetic contribution and the weight were estimated by comparing the magnetization vs. magnetic field at $5 \mathrm{~K}$ and $\chi \mathrm{T}$ curves measured in the $2-300 \mathrm{~K}$ before irradiation with those recorded for the sample in a routine experiment $(22,34 \mathrm{mg}$ of sample loaded into gel caps, diamagnetism contribution calculated with Pascal constants at $\left.\chi_{\text {dia }}=-450010^{-6} \mathrm{~cm}^{3} \mathrm{~mol}^{-1}\right)$. These comparisons give $\mathrm{m}$ $=3 \mathrm{mg}$ and diamagnetic correction $=-7.610^{-3} \mathrm{emu} \mathrm{mol}^{-1}$ (experiment A), and $5 \mathrm{mg}$ and $-8.410^{-3} \mathrm{emu} \mathrm{mol}^{-1}$ (experiment B). The nanoparticules were irradiated continuously using the multiline 406-415 $\mathrm{nm}$ under a magnetic field at $5 \mathrm{kOe}$ at $10 \mathrm{~K}$, till the magnetization saturation was obtained (14 hours experiment A et 17 hours experiment B). The power laser light received by the sample was $7 \mathrm{~mW} / \mathrm{cm}^{2}$.

For the microSQUID measurements, only few microgramms are used and the fiber is only $1 \mathrm{~cm}$ away from the sample 
Figures

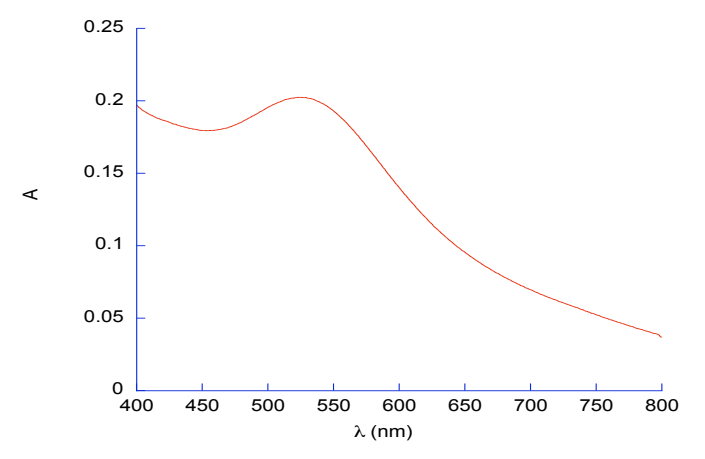

Fig. S1. UV-vis absorption spectrum of CuNiMo $(\mathrm{CN})_{8}(\mathrm{pyr})_{47} .59 \mathrm{H}_{2} \mathrm{O}$ particles in water $\left(5.10^{-4} \mathrm{M}\right)$.

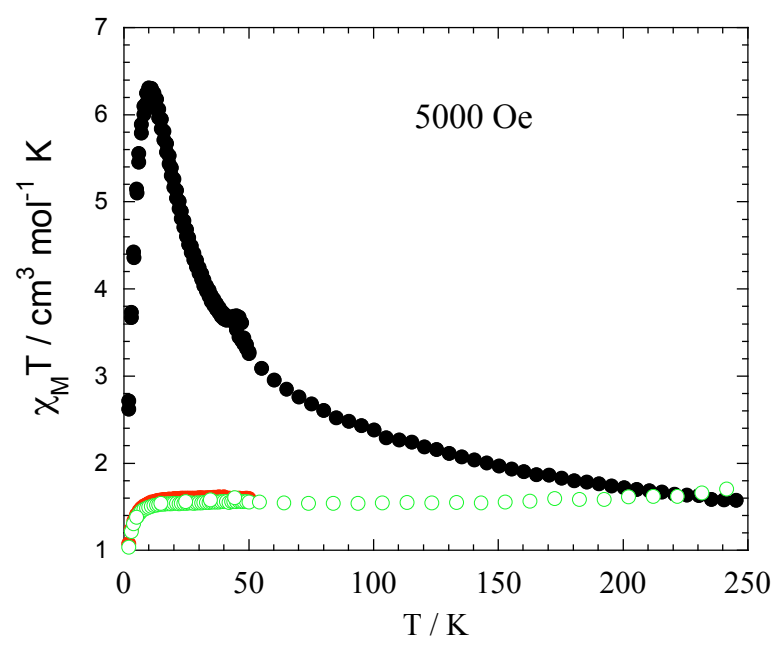

Fig. S2. $\chi \mathrm{T}=\mathrm{f}(\mathrm{T})$ observed before irradiation $(\bigcirc)$, after irradiation at $\mathrm{T}=10 \mathrm{~K}(\bigcirc)$, and after thermal relaxation $(\mathrm{O})$ at $\mathrm{T}$ $=300 \mathrm{~K}$.

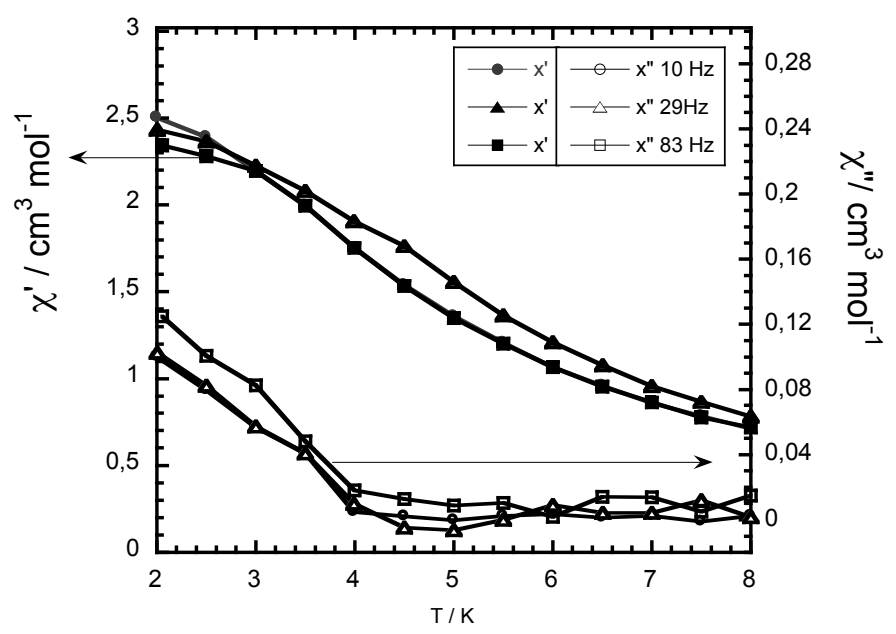

Fig. S3. $\chi{ }^{\prime}$ and $\chi \chi^{\prime}=\mathrm{f}(\mathrm{T})$ after irradiation 


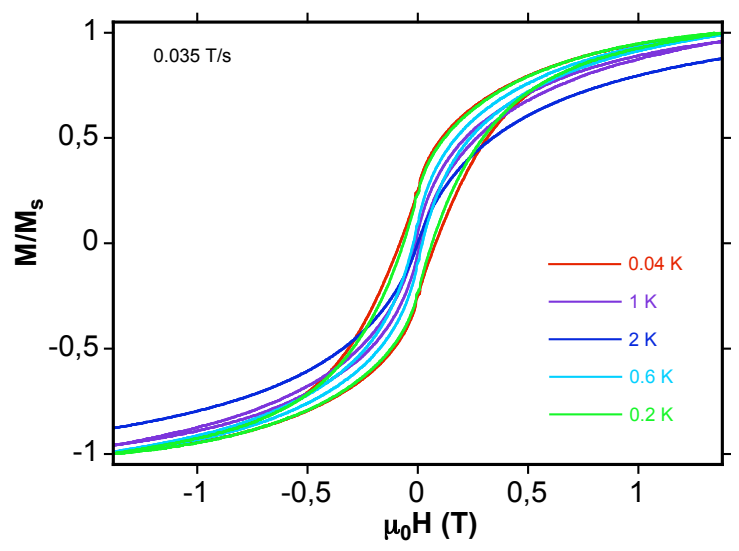

Fig. S4 $\mathrm{M}=\mathrm{f}\left(\mu_{0} \mathrm{H}\right)$ at different temperatures after irradiation

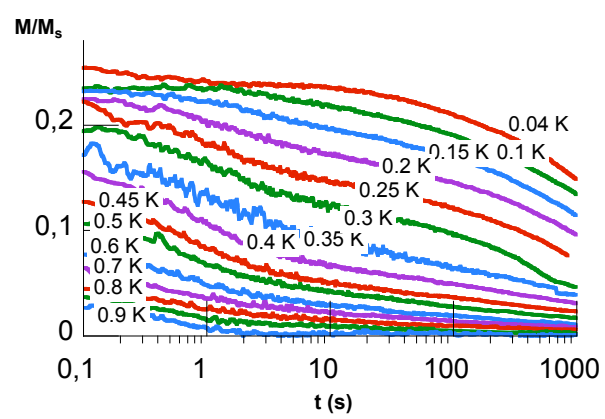

Fig. S5 Magnetization decay versus time.

In order to extract $\tau_{0}$ for each temperature, one method consists in scaling the magnetization $v s$. time so that all the data fall on one master curve. In such a case, $\tau$ for each temperature is proportionnal to the inverse of the scaling factor. Figure S6 shows actually that all the data fall on one curve. Since we deal with particles that are not strictly identical, it is expected that several relaxation processes are present at each temperature. The smaller particles have shorter relaxation time than the larger ones. This means as well that we have a distribution of energy barriers. In order to remove the contribution of the smaller particles we neglected the data points of figure S5 corresponding to short relaxations. The scaling of the data was possible as shown in Fig. S6 and all fall on one master curve. The quality of the curve is very good for a set of nanoparticles. From the scaling factors obtained one can plot Fig. S7 and extract $\tau_{0}$ and $\mathrm{E}_{\mathrm{a}}$ the energy barrier at zero field corresponding to the thermal activated relaxation regime.

It is clear from Figure $\mathrm{S} 7$ that below $\mathrm{T}=0.3 \mathrm{~K}$, the relaxation is not thermally activated but rather temperature independent which corresponds to a tunneling relaxation regime.

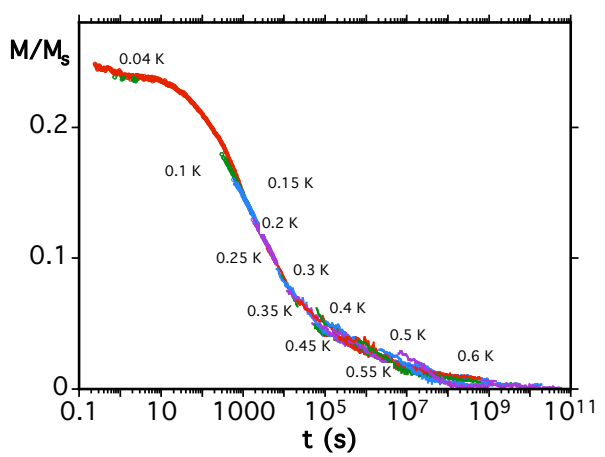

Figure S6. Scaled magnetization $v s$. time at different tempertaures 


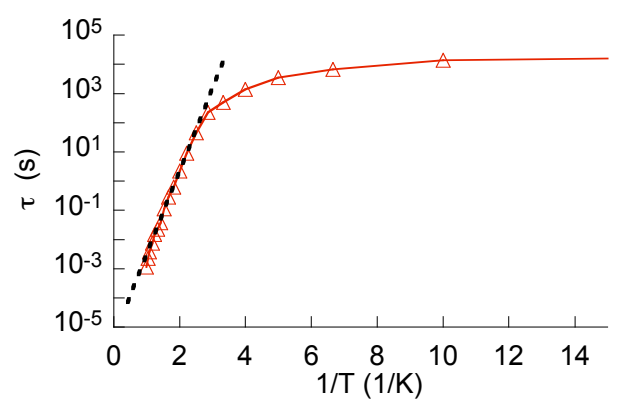

Fig. S7 Thermal dependence of the relaxation time (logarithmic scale for $\tau$ ). The linear dependence can be fitted by an Arrhenius law (dashed line) $\tau=3.10^{-6} \exp (6.7 / \mathrm{T})$.

Method applied to study the reversal mechanism of the magnetization :

For a Neel-Brown Arrhenius law, the coercitive field can be expressed as $H_{c}(T, v)=H_{c}^{0}\left(1-\left(k T / E_{a}\right) \ln (c / v)\right)^{1 / \alpha}(\mathbf{1})$ where $v$ $=\mathrm{dH} / \mathrm{dt}$ is the field sweeping rate and $\mathrm{c}=\mathrm{H}_{\mathrm{c}}{ }^{0} k \mathrm{~T} /\left(2 \tau_{0} \mathrm{E}_{\mathrm{a}}\left(1-\mathrm{H}_{\mathrm{c}} / \mathrm{H}_{\mathrm{c}}{ }^{0}\right)\right)$ with $\mathrm{H}_{\mathrm{c}}{ }^{0}$ the mean reversal field of an identical assembly of nanoparticles at zero temperature, $\mathrm{E}_{\mathrm{a}}$ the energy barrier at zero applied field and $\alpha$ a constant comprised between 1.5 and 2 (this model is an approximation since the nanoparticles are not strictly identical even though they have a very weak size distribution). We will assume as well $\alpha=2$ as usually for single-domain particles with uniaxial anisotropy.

If the nanoparticles follow the model expressed by eq. 1 , the data corresponding to the plots $\mathrm{H}_{\mathrm{c}}(\mathrm{T}, \mathrm{v}) \mathrm{vs}[\mathrm{T} \ln (\mathrm{c} / \mathrm{v})]^{1 / 2}$ at different temperatures must fall on one master curve for a given value of $\tau_{0}$ (Fig. S10)

A convenient method to do so is to, first, register the temperature dependence of the coercitive field at various sweep field rates $v$ (Fig S8 and S9). For a thermally activated process, the coercive field decreases upon heating and when the sweep rate is decreased. This is what we observe (Fig. S8 and S9).

The second step consists of plotting the energy barrier $\Delta \mathrm{E} v s$. the coercive field $\mu_{0} \mathrm{H}_{\mathrm{c}}(\operatorname{since} \Delta \mathrm{E}=\mathrm{kT} \ln (\mathrm{c} / v)$ which leads to $\left.\Delta \mathrm{E}=\mathrm{E}_{\mathrm{a}}\left[1-\left(\mathrm{H}_{\mathrm{c}} / \mathrm{H}_{\mathrm{c}}^{0}\right)^{2}\right]\right)$ that allows to determine the value of the energy barrier $\left(\mathrm{E}_{\mathrm{a}}\right)$ at zero field (Fig. S11) and compare it to that obtained from the scaling of the magnetization (see above). A proof that the nanoparticles follow the model we assumed is given if the two $\mathrm{E}_{\mathrm{a}}$ values have the same magnitude.

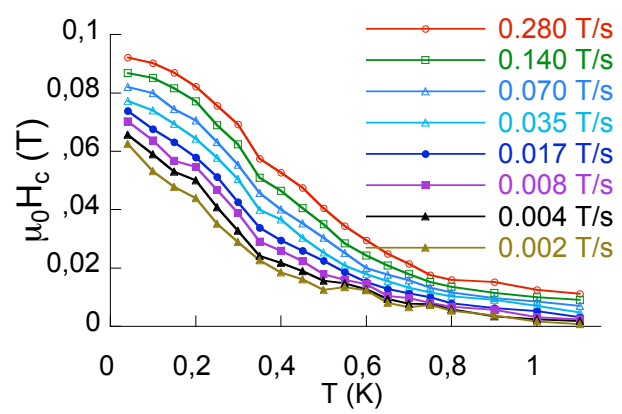

Fig. S8 Coercitive field as a function of the temperature at several field sweep rates.

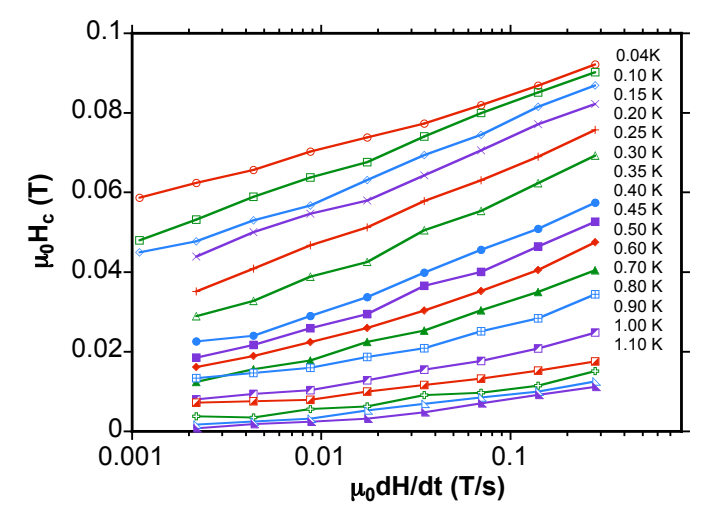

Fig. S9 Coercitive field dependence of the weep field rate at various temperatures. 


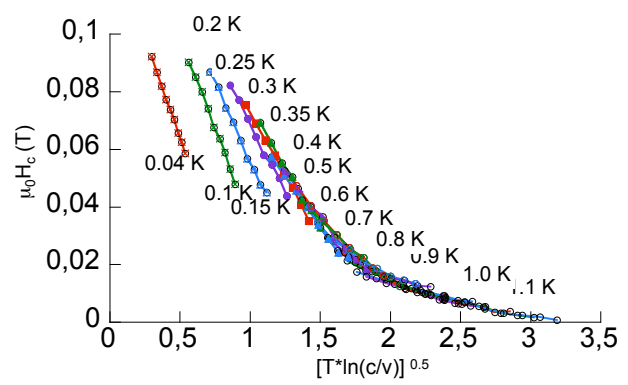

Fig. S10 Scaling plot of the coercitive field $\mathrm{H}_{c}(\mathrm{~T}, v)$ for field sweep rates between $0.002 \mathrm{~T} / \mathrm{s}$ and $0.280 \mathrm{~T} / \mathrm{s}$ and different temperatures assuming the $\tau_{0}$ value found from figure $\mathrm{S} 7$

The fact that all the points fall on a master curve for $\mathrm{T}>0.3 \mathrm{~K}$ indicates that the behaviour of these particles can be described by (1). Below $0.3 \mathrm{~K}$, the data do not fall on the master curve : this suggests the occurrence of quantum tunneling.

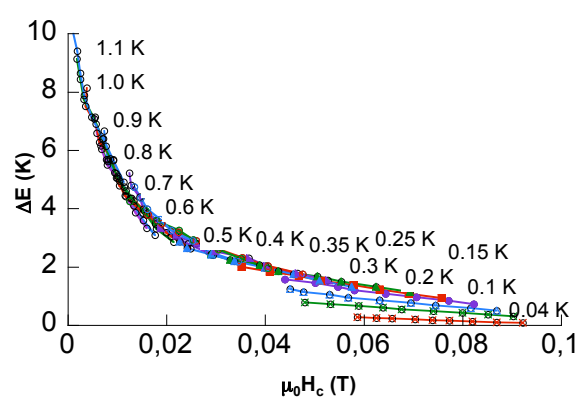

Fig. S11 Field dependence of the energy barrier of the particles at different tempertaures.

From Fig. $\mathrm{S} 11$ one can see that $\Delta \mathrm{E}$ at zero field $\left(\mathrm{E}_{\mathrm{a}}\right)$ is around $10 \mathrm{~K}$ which is in good agreement with the energy barrier value extracted from Fig. S6 equal to $6.7 \mathrm{~K}$.

The fact that at low temperatures the data do not fall on the same plot as for the higher tempertaures is indicative that the relaxation process is not thermally activated and suggests the occurrence of quantum tunneling of the magnetization.

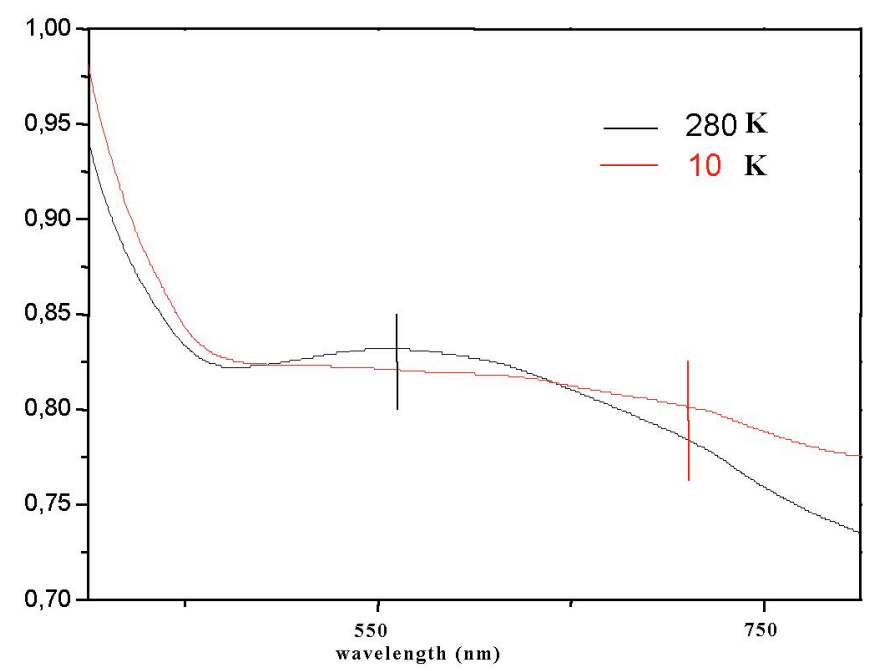

Fig. S12 Reflectivity spectra in the UV-visible region at $280 \mathrm{~K}$ (black) and $10 \mathrm{~K}$ (red) after irradiation of the sample 